\title{
The Local Recurrence of Breast Cancer with Squamous Metaplasia and Obvious Histological Heterogeneity
}

\author{
NAMI YAMASHITA ${ }^{1}$, ERIKO TOKUNAGA ${ }^{2}$, HIDETAKA YAMAMOTO $^{3}$, CHIKAKO SHIMIZU $^{4}$, \\ KENJI TAKETANI $^{1}$, YUKA INOUE ${ }^{1}$, HIROSHI SAEKI ${ }^{1}$, EIJI OKI $^{1}$ and YOSHIHIKO MAEHARA ${ }^{1}$ \\ ${ }^{1}$ Department of Surgery and Science, ${ }^{3}$ Department of Anatomic Pathology, Pathological Science, \\ Graduate School of Medical Sciences, Kyushu University, Fukuoka, Japan; \\ ${ }^{2}$ Department of Breast Oncology, National Hospital Organization Kyushu Cancer Center, Fukuoka, Japan; \\ ${ }^{4}$ Department of Breast and Medical Oncology, National Cancer Center Hospital, Tokyo, Japan
}

\begin{abstract}
Case Report: We herein report a case of local recurrence of breast cancer with squamous metaplasia and obvious intratumoral and intertumoral heterogeneity. A 39year-old female patient was diagnosed with T3N2MO stage $I I I B$ right breast cancer and underwent right total mastectomy and axillar lymph node dissection. At four years after surgery, she became aware of chest wall pain and diagnostic imaging revealed recurrence in the lung, right thoracic wall and sternum. The recurrent lesions remained stable for 18 months with endocrine therapy. Thereafter, the lesion in the right thoracic wall suddenly became enlarged. Moreover, liver metastasis was confirmed on FDG-PET/CT. She underwent right thoracic wall tumor resection. A biopsy was simultaneously performed to obtain a specimen from the site of liver metastasis. Postoperatively, the right chest wall mass showed obvious intratumoral heterogeneity; squamous differentiation with aggressive features and a papillotubular component similar to the primary tumor. The metastatic liver tumor showed similar pathological features to the primary tumor. Conclusion: Intratumoral and intertumoral heterogeneity within primary tumors and associated metastatic sites may contribute to treatment failure and drug resistance.
\end{abstract}

Intratumoral and intertumoral heterogeneity has been reported in primary human solid tumors and is derived from

Correspondence to: Prof. Yoshihiko Maehara, Department of Surgery and Science, Graduate School of Medical Sciences, Kyushu University, 3-1-1 Maidashi, Higashi-ku, Fukuoka 812-8582, Japan. Tel: +81 926425466,Fax: +81926415482,e-mail: maehara@ surg2.med.kyushu-u.ac.jp

Key Words: Breast cancer, local recurrence, squamous metaplasia, intratumoral heterogeneity, intertumoral heterogeneity. the interplay between genetic and non-genetic factors (1-4). Intratumoral and intertumoral heterogeneity within primary tumors and associated metastatic sites may contribute to treatment failure and drug resistance, and may therefore lead to difficulties in clinical management. We herein report the case of a patient who showed local recurrence of breast cancer with squamous metaplasia and obvious intratumoral and intertumoral heterogeneity. Written informed consent was obtained from the patient for the publication of this case report and any accompanying images.

\section{Case Presentation}

Clinical course. A 39-year-old premenopausal female patient was admitted to the Kyushu University Hospital with right breast cancer in 2006. The tumor was a palpable mass of 55 $\mathrm{mm}$ in diameter in the inner-upper area of the right breast; direct invasion to the pectoralis major was suspected. A core needle biopsy specimen from the tumor showed invasive ductal carcinoma. Immunohistochemically, the tumor was positive for estrogen receptor (ER) (Allred: proportion score (PS) 4 + intensity score (IS)2=total score (TS)6, progesterone receptor (PR) (Allred: PS4+IS3=TS7) and negative for human epidermal growth factor receptor 2 (HER2) (score $1+)$. Based on the clinical findings, the clinical stage was diagnosed as T2N1M0 stage IIIB, according to the UICC TNM classification of malignant tumors. The patient received neoadjuvant chemotherapy consisting of four courses of 5fluorouracil $\left(500 \mathrm{mg} / \mathrm{m}^{2}\right)$, epirubicin $\left(75 \mathrm{mg} / \mathrm{m}^{2}\right)$, and cyclophosphamide $\left(500 \mathrm{mg} / \mathrm{m}^{2}\right)$. The size of the breast tumor did not decrease and she underwent right total mastectomy and axillar lymph node dissection. The histological diagnosis, according to The Japanese Breast Cancer Society's General Rules for Clinical and Pathological Recording of Breast Cancer (ref), was invasive ductal carcinoma (scirrhous/ papillotubular carcinoma; invasive size, $5.5 \mathrm{~cm}$; nuclear 
grade, 3 [nuclear atypia 3+ mitotic count 3]), and was negative for lymphovascular invasion and vessel invasion. Carcinoma cells were detected in four lymph nodes. Immunohistochemically, the tumor was positive for ER (PS4+IS3=TS7), PR (PS2+IS3=TS5) and negative for HER2 (score 1+). The patient received four courses of docetaxel $\left(75 \mathrm{mg} / \mathrm{m}^{2}\right)$ as adjuvant chemotherapy, radiation therapy for the right chest wall (50 Gy/25 Fr+Boost $10 \mathrm{~Gy} / 5 \mathrm{Fr}$ ), supraclavicular and internal thoracic areas (50 Gy/25 Fr), followed by endocrine therapy (goserelin and tamoxifen). At four years after the operation, she became aware of right chest wall pain and diagnostic imaging revealed recurrence in the lung, right thoracic wall and sternum. The recurrent lesions became stable for 18 months after changing the endocrine therapy to goserelin and letrozole.

Thereafter, the lesion in the right thoracic wall suddenly showed rapid growth (Figure 1A). The mass grew from 5 $\mathrm{mm}$ to $40 \mathrm{~mm}$ within one month. A core needle biopsy (CNB) of the mass revealed the proliferation of carcinoma cells with eosinophilic cytoplasm arranged in solid or nested patterns with keratinization and necrosis (Figure 1B). The pathological findings were quite different from those of the postoperative specimen. No scirrhous or papillotubular carcinoma were seen. The tumor nest with squamous differentiation was immunohistochemically negative for ER, PR, and HER2. A CT scan showed that a heterogeneously enhanced mass on the right thoracic wall rapidly enlarged from $5 \mathrm{~mm}$ to $40 \mathrm{~mm}$ within a month (Figures $2 \mathrm{~A}$ and $\mathrm{B}$ ). An FDG-PET/CT scan showed a mass in the right thoracic wall $\left(\mathrm{SUV}_{\max }=23.5\right)$ and liver masses $\left(\mathrm{SUV}_{\max }=8.5\right)$ in segment 4 of the left liver lobe (Figure 2C). An FDGPET/CT scan showed no evidence of squamous cell carcinoma (SCC) at any other primary sites, including the lung, esophagus and vagina. The punch biopsy specimen of the skin immediately above the mass showed no evidence of SCC of the skin. We diagnosed the mass as a local recurrence of the breast tumor. The right chest wall tumor was resected and repaired by a skin graft.

At four weeks after surgery, a fast growing subcutaneous nodule appeared in the left edge of the skin graft again. Radiation therapy and the administration of capecitabine was started in order to treat local recurrence, multiple liver, lung and bone metastasis. The mass in the left thoracic wall gradually shrunk. However, multiple liver metastases were found to have become progressive disease at two months. Despite changing the regimen to oral combination chemotherapy with capecitabine and cyclophosphamide, the liver and lung metastases became uncontrolled within one month. The disease reached life-threatening stage, and combination therapy with paclitaxel and bevacizumab was subsequently initiated. Thereafter, the patient was treated with eribulin and vinorelbine monotherapy; however, the disease progressed rapidly. In addition to uncontrolled multiple lung and liver metastases, multiple brain metastases appeared. She died of breast cancer 3 years and 2 months after the recurrence.

Pathological findings. A macroscopic examination of the metastatic tumor on the right chest wall revealed a solid, white lesion of $38 \mathrm{~mm} \times 30 \mathrm{~mm}$ in size, with an extensive area of central necrosis (Figure 3A). A histological analysis revealed that the tumor was composed of two distinct components: a squamous component with aggressive features, and a complex grandular growth pattern (Figure 3B). The former component occupied the pectoral side and the marginal part of the tumor, while the carcinoma cells proliferated in a tubular, grandular or papillary pattern (Figure 3C). Immunohistochemically, this area was positive for ER (PS2+IS1=TS3, 2.5\%), PR (PS2+IS3=TS5, 5\%), focally positive for $\mathrm{p} 63$ and negative for HER2 (score 1+). The MIB-1 labelling index was $10 \%$ (Figures 4A-E, left). In the latter component, which occupied the center of the tumor, the carcinoma cells proliferated in a solid pattern, accompanied by hemorrhage, necrosis and focal squamous differentiation with occasional keratinization (Figure 3D). Immunohistochemically, this area was positive for p63, but negative for ER (PS0+IS0=TS0, 0\%), PR (PS0+IS0=TS0, 0\%) and HER2 (score 0). The MIB-1 labelling index was $65 \%$ (Figure 4A-E, right side). The features indicated a recurrence of breast carcinoma accompanied by squamous differentiation.

This case also showed intertumoral heterogeneity. The primary tumor was invasive ductal carcinoma with a complex glandular growth pattern (Figure 3E). The right thoracic wall mass showed obvious intratumoral heterogeneity, a squamous component with aggressive features and a complex glandular growth pattern similar to the primary tumor. A simultaneously obtained CNB specimen of the metastatic liver tumor showed the proliferation of carcinoma cells arranged in a sinusoidal or nested pattern without keratinization, which was pathologically similar to the primary tumor (Figure 3F).

\section{Discussion}

We report a case of the local recurrence of breast cancer with squamous metaplasia and obvious intratumoral and intertumoral heterogeneity.

In less than $5 \%$ of mammary adenocarcinomas, part or all of the carcinomatous epithelium is transformed into a nonglandular growth pattern. The process is referred to as metaplasia. Metaplastic carcinoma of the breast is a rare and aggressive breast cancer phenotype, which usually expresses very low levels of ER, PR and HER2 (5-7). Unlike other triple-negative breast cancers, it seems that metaplastic breast carcinomas do not respond to conventional chemotherapy regimens (8). The term, "metaplastic breast carcinoma" encompasses a heterogeneous group of tumors 

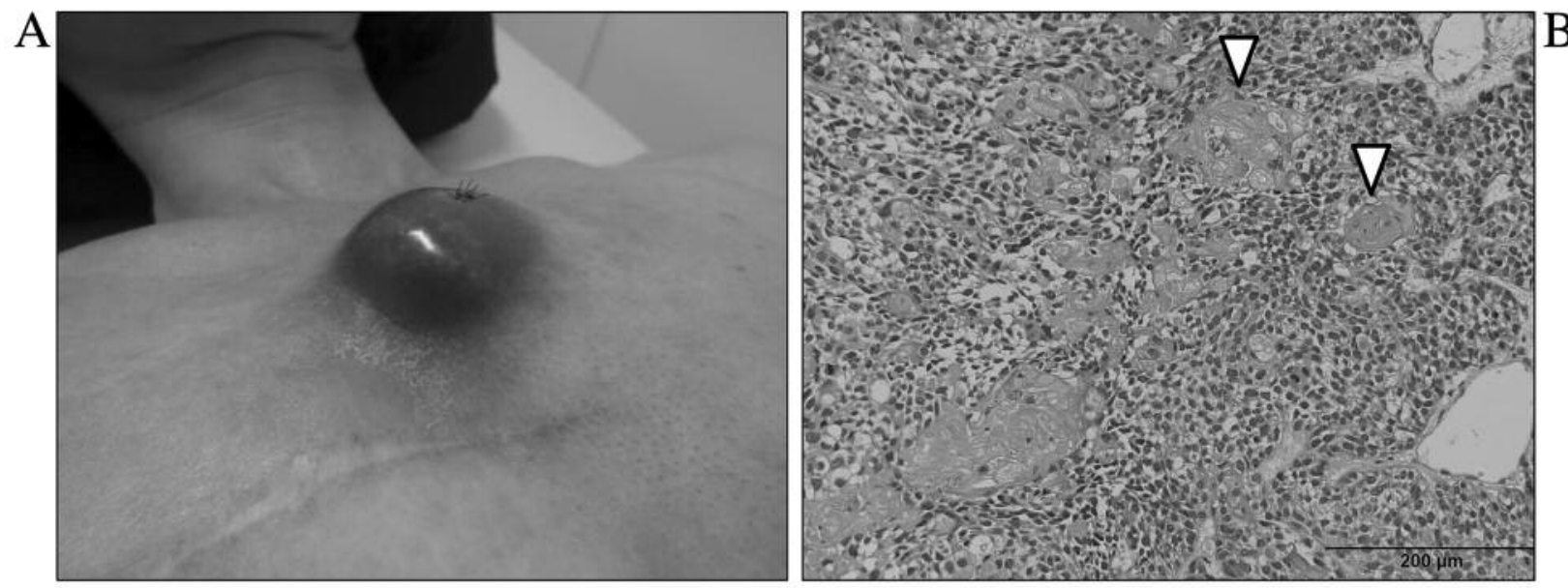

Figure 1. The physical appearance of the tumor and hematoxylin \& eosin staining of the CNB specimen. (A) A rapidly enlarged lesion in the right thoracic wall. The mass grew in size from $5 \mathrm{~mm}$ to $40 \mathrm{~mm}$ within one month. (B) The histological findings included the proliferation of carcinoma cells arranged in solid or nested patterns with keratinization (arrows) and necrosis (Original magnification, ×200). H\&E: Hematoxylin and eosin; CNB: core needle biopsy.
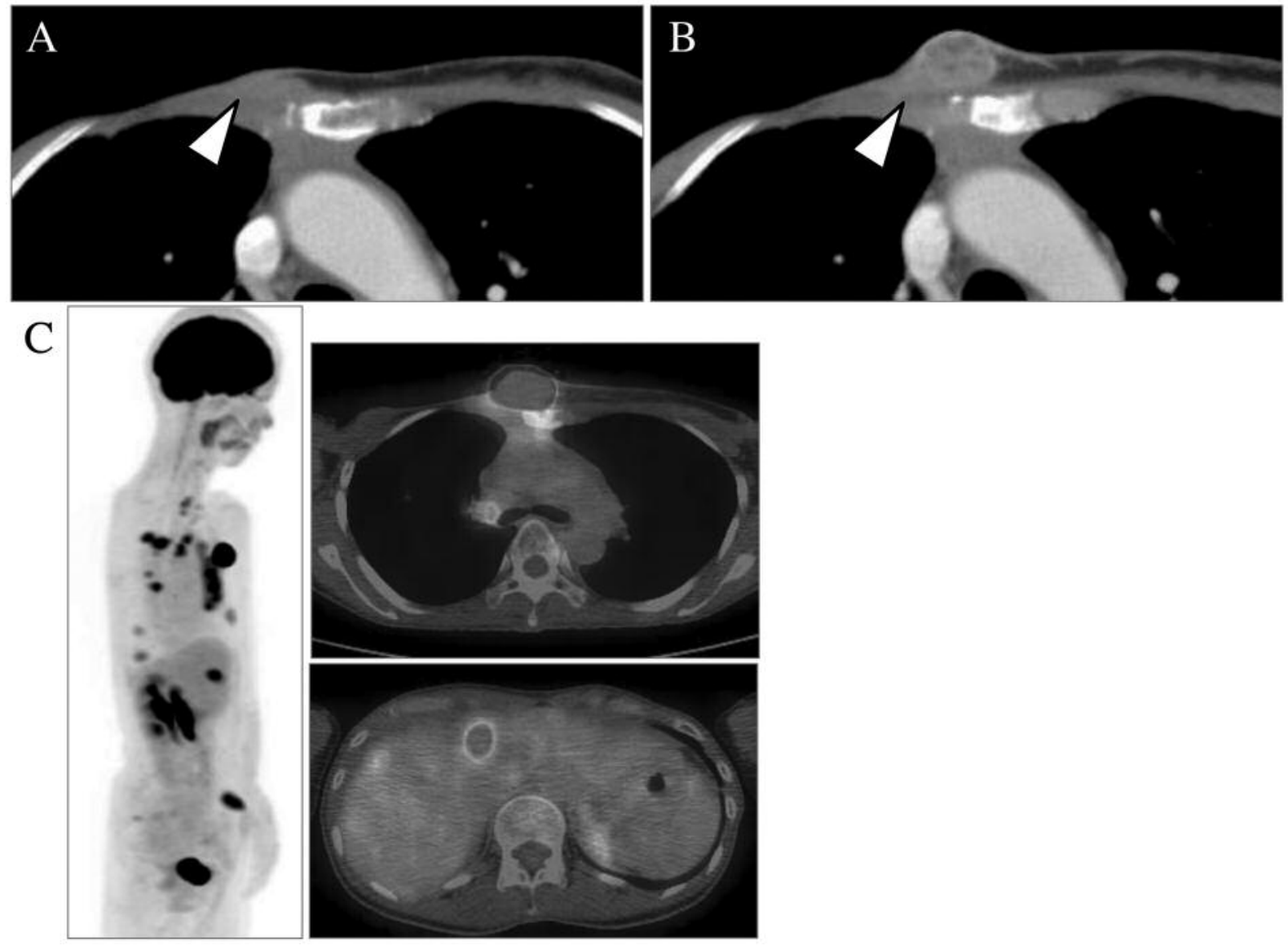

Figure 2. Diagnostic imaging. (A, B) A CT scan showed a heterogeneously enhanced mass on the right thoracic wall that rapidly grew from 5 mm to $40 \mathrm{~mm}$ within one month (arrowheads). (C) An FDG-PET/CT scan showed a right thoracic wall mass (SUVmax=23.5) and liver masses (SUVmax=8.5) in segment 4 of the left liver lobe. There was no evidence of primary squamous cell carcinoma (SCC) at any other sites, including the lung, esophagus or vagina. CT: Computed tomography; MRI: magnetic resonance imaging; FDG-PET: fluorodeoxyglucose-positron emission tomography. 

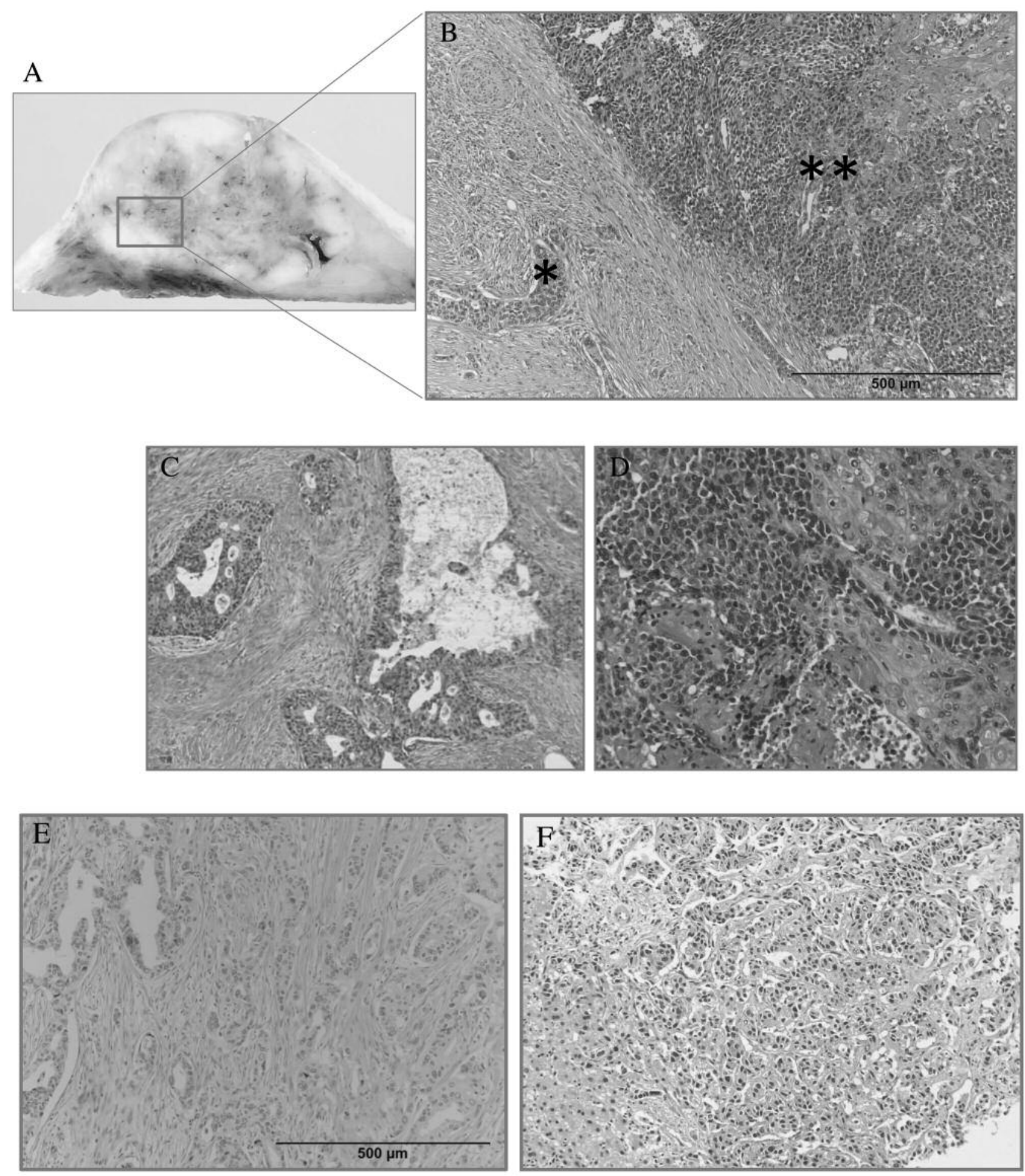

Figure 3. The gross appearance and pathological findings. (A) The circumscribed mass was solid and white, measuring $38 \mathrm{~mm} \times 30 \mathrm{~mm}$ in size, with an extensive area of central necrosis. (B) A low-power view of $H \& E$ staining showed that the tumor composed of two distinct components: a papillotubular-like component $(*)$ and a solid component $(* *) .(C)$ The papillotubular-like component $(*)$ occupied the pectoral side and marginal part of the tumor, carcinoma cells proliferated in tubular, glandular, or papillary pattern. (D) The solid component (**) occupied the center of the tumor; carcinoma cells proliferated in a solid pattern, accompanied by hemorrhage, necrosis and focal squamous differentiation with occasional keratinization. A comparison of $H \& E$ staining of the primary tumor $(E)$ and the site of metastasis $(F)$. Both histological findings showed invasive ductal carcinoma with a tubular, glandular, or papillary growth pattern. (C-F; original magnification, $\times 200)$. 

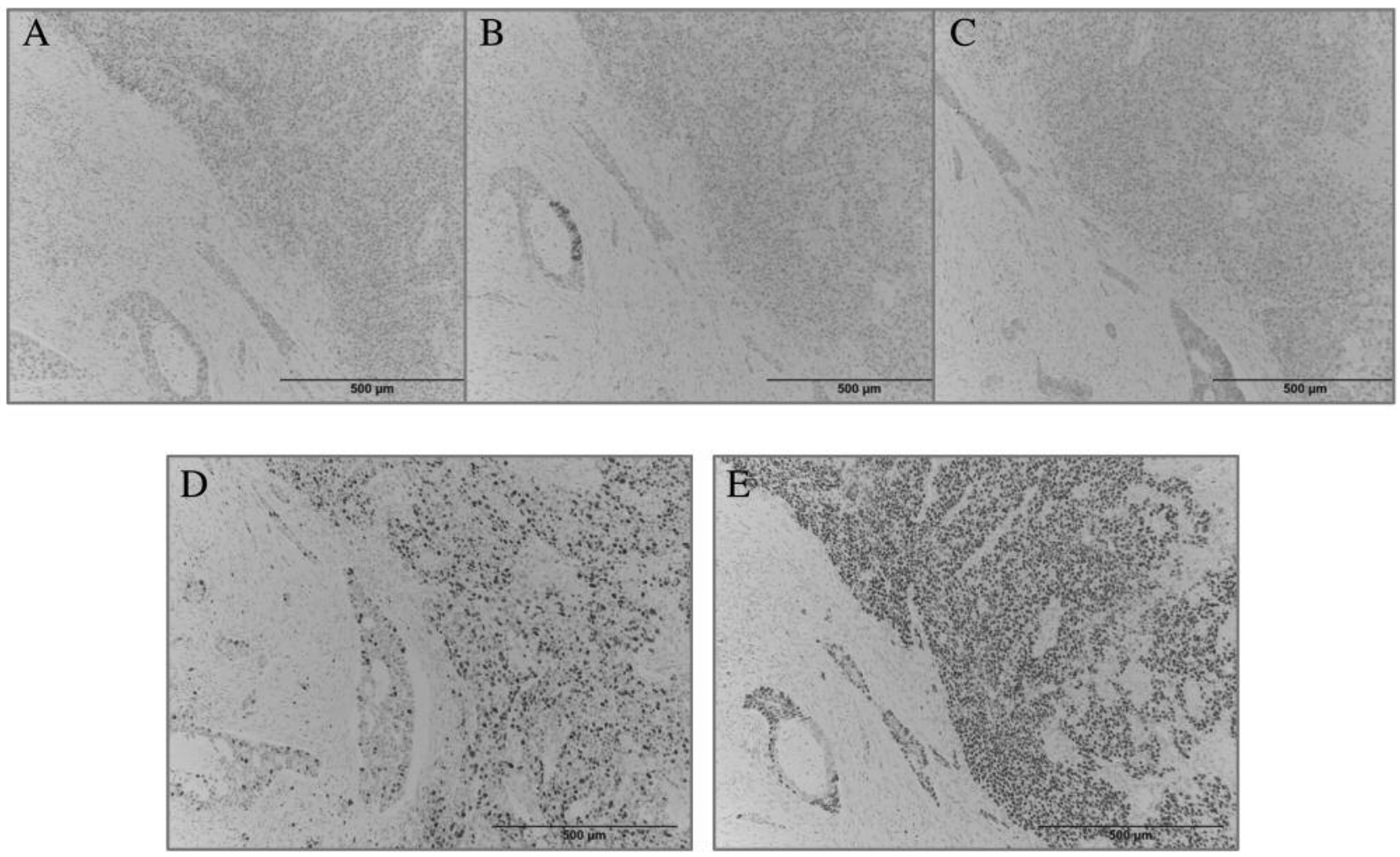

Figure 4. Immunohistochemical staining for ER, PR, HER2, Ki67 and p63. (A) ER, (B) PR, (C) HER2, (D) Ki67 and (E) p63. Left, the papillotubularlike component was positive for ER 2.5\%, PR 5\%, focally positive for p63 and negative for HER2 (score 1+). The MIB-1 labelling index was $10 \%$. Right, the solid component was positive for p63 but negative for ER 0\%, PR 0\% and HER2 (score 0). The MIB-1 labelling index was 65\% (original magnification, $\times 100$ ).

characterized by the presence of malignant cells, showing differentiation towards squamous epithelium or chondroid, as well as osseous and rhabdoid differentiation $(9,10)$.

Squamous metaplasia was reportedly present in $3.7 \%$ of 1,665 invasive carcinomas reviewed by Fisher et al. (11). The precise cell type that gives rise to metaplastic carcinoma remains uncertain. Some studies have reported the presence of clonality in the epithelial and metaplastic components, which indicates that they originated from a single stem cell $(12,13)$. Others have reported that the metaplastic components had an epithelial (14) or myoepithelial (15) origin. In our case, the concurrent presence of the invasive ductal carcinoma component and the transitions observed from the carcinomatous foci to squamous metaplasia indicated that these neoplasms were derived from mammary epithelial cells. Immunohistochemical studies showed stronger p63, involculin (data not shown) and p53 staining (data not shown) in metaplastic components, suggesting that these cells have myoepithelial features and squamous differentiation.

Tumor cells often display remarkable diversity, including clinically important phenotypes (i.e., to seed metastases and acquire resistance to therapeutic agents). Our case showed a poor response to neoadjuvant chemotherapy and the recurrence occurred during adjuvant endocrine therapy. The tumor might have initially displayed drug resistance and also acquired multidrug resistance during the clinical course. It is important to note that phenotypic heterogeneity is not determined solely through genetic diversity between subclones, it is also influenced by varied patterns of proliferation, and epigenetic divergence with dormant resting cells surviving cytotoxic exposure (16-18). Close inspection and an understanding of intratumoral and intertumoral heterogeneity will be useful for developing targeted therapies and future therapeutic approaches to limit cancer diversity, adaptation and drug resistance.

\section{Conclusion}

We reported a case of local recurrence of breast cancer with squamous metaplasia and obvious intratumoral and intertumoral heterogeneity. Tumor heterogeneity within primary tumors and associated metastatic sites may contribute to treatment failure and drug resistance. 


\section{Conflicts of Interest}

The Authors declare no conflicts of interest in association with the present study.

\section{References}

1 Gerlinger M, Rowan AJ, Horswell S, Math M, Larkin J, Endesfelder D, Gronroos E, Martinez P, Matthews N, Stewart A, Tarpey P, Varela I, Phillimore B, Begum S, McDonald NQ, Butler A, Jones D, Raine K, Latimer C, Santos CR, Nohadani M, Eklund AC, Spencer-Dene B, Clark G, Pickering L, Stamp G, Gore M, Szallasi Z, Downward J, Futreal PA and Swanton C: Intratumor heterogeneity and branched evolution revealed by multiregion sequencing. N Engl J Med 366: 883-892, 2012.

2 Anderson K, Lutz C, van Delft FW, Bateman CM, Guo Y, Colman SM, Kempski H, Moorman AV, Titley I, Swansbury J, Kearney L, Enver T and Greaves M: Genetic variegation of clonal architecture and propagating cells in leukaemia. Nature 469: 356-361, 2011.

3 Campbell LL and Polyak K: Breast tumor heterogeneity: cancer stem cells or clonal evolution? Cell Cycle 6: 2332-2338, 2007.

4 Shackleton M, Quintana E, Fearon ER and Morrison SJ: Heterogeneity in cancer: cancer stem cells versus clonal evolution. Cell 138: 822-829, 2009.

5 Wargotz ES and Norris HJ: Metaplastic carcinomas of the breast. IV. Squamous cell carcinoma of ductal origin. Cancer 65: 272276, 1990.

6 Wargotz ES and Norris HJ: Metaplastic carcinomas of the breast: V. Metaplastic carcinoma with osteoclastic giant cells. Hum Pathol 21: 1142-1150, 1990 .

7 Weigelt B, Geyer FC and Reis-Filho JS: Histological types of breast cancer: how special are they? Mol Oncol 4: 192-208, 2010.

8 Hennessy BT, Giordano S, Broglio K, Duan Z, Trent J, Buchholz TA, Babiera G, Hortobagyi GN and Valero V: Biphasic metaplastic sarcomatoid carcinoma of the breast. Ann Oncol 17: 605-613, 2006.

9 Lakhani SR, Ellis IO and Schnitt SJ: Special subtypes. In: WHO Classification of Tumours of the Breast, 4th edn. IARC Press, Lyon. pp. 39-76, 2012.
10 Weigelt B, Eberle C, Cowell CF, Ng CK, Reis-Filho JS. Metaplastic breast carcinoma: more than a special type. Nat Rev Cancer 14: 147-148, 2014.

11 Fisher ER, Palekar AS, Gregorio RM and Paulson JD: Mucoepidermoid and squamous cell carcinomas of breast with reference to squamous metaplasia and giant cell tumors.Am J Surg Pathol 7: 15-17, 1984

12 Wada H, Enomoto T, Tsujimoto M, Nomura T, Murata Y and Shroyer KR: Carcinosarcoma of the breast: molecular-biological study for analysis of histogenesis. Hum Pathol 29: 1324-1328, 1998.

13 Teixeira MR, Qvist H, Bohler PJ, Pandis N and Heim S: Cytogenetic analysis shows that carcinosarcomas of the breast are of monoclonal origin. Genes Chromosomes Cancer 22: 145$151,1998$.

14 Palmer JO, Ghiselli RW and McDivitt RW: Immunohistochemistry in the differential diagnosis of breast diseases. Pathol Annu 25 Pt 2: 287-315, 1990.

15 Wargotz ES and Norris HJ: Metaplastic carcinomas of the breast. III. Carcinosarcoma. Cancer 64: 1490-1499, 1989.

16 Marusyk A, Almendro $\mathrm{V}$ and Polyak K: Intra-tumour heterogeneity: a looking glass for cancer? Nat Rev Cancer 12: 323-334, 2012.

17 Meads MB, Gatenby RA and Dalton WS: Environment-mediated drug resistance: a major contributor to minimal residual disease. Nat Rev Cancer 9: 665-674, 2009.

18 Kreso A, O’Brien CA, van Galen P, Gan OI, Notta F, Brown AM, Ng K, Ma J, Wienholds E, Dunant C, Pollett A, Gallinger S, McPherson J, Mullighan CG, Shibata D and Dick JE: Variable clonal repopulation dynamics influence chemotherapy response in colorectal cancer. Science 339: 543-548, 2013. 